\title{
Association of Sickle Cell Trait with Risk and Mortality of COVID-19: Results from the United Kingdom Biobank
}

\author{
W. Kyle Resurreccion, ${ }^{1}$ Joseph Hulsizer, ${ }^{2}$ Zhuqing Shi, ${ }^{1}$ Jun Wei, ${ }^{1}$ Chi-Hsiung Wang, ${ }^{1}$ Rong Na, ${ }^{1}$ S. Lilly Zheng, ${ }^{1}$ Clay Struve, ${ }^{3}$ \\ Brian T. Helfand, ${ }^{1,4}$ Janardan Khandekar, ${ }^{5}$ Liana K. Billings, ${ }^{6,7}$ Michael S. Caplan, ${ }^{8}$ and Jianfeng Xu ${ }^{1,4 *}$ \\ ${ }^{1}$ Program for Personalized Cancer Care, NorthShore University HealthSystem, Evanston, Illinois; ${ }^{2}$ Lake Forest Academy, Lake Forest, Illinois; \\ ${ }^{3}$ CSS LLC, Chicago, Illinois; ${ }^{4}$ Department of Surgery, University of Chicago Pritzker School of Medicine, Chicago, Illinois; ${ }^{5}$ Center for Molecular \\ Medicine, NorthShore University HealthSystem, Evanston, Illinois; ${ }^{6}$ Department of Medicine, NorthShore University HealthSystem, Evanston, \\ Illinois ${ }^{7}$ Department of Medicine, University of Chicago Pritzker School of Medicine, Chicago, Illinois; ${ }^{8}$ Department of Pediatrics, NorthShore \\ University HealthSystem, Evanston, Illinois
}

\begin{abstract}
Sickle cell trait (SCT) carriers inherit one copy of the Glu6Val mutation in the hemoglobin gene and is particularly common in Black individuals (5-10\%). Considering the roles of hemoglobin in immune responses and the higher risk for coronavirus disease (COVID-19) among Black individuals, we tested whether Black SCT carriers were at increased risk for COVID-19 infection and mortality according to the United Kingdom Biobank. Among Black individuals who were tested for COVID-19, we found similar infection rates among SCT carriers $(14 / 72 ; 19.7 \%)$ and noncarriers $(167 / 791 ; 21.1 \%)$, but higher COVID-19 mortality rates among SCT carriers $(4 / 14 ; 28.6 \%)$ than among noncarriers $(21 / 167 ; 12.6 \%)$ (odds ratio [OR], 3.04; 95\% confidence interval $[\mathrm{Cl}], 0.69-11.82 ; P=0.12)$. Notably, SCT carriers with preexisting diabetes had significantly higher COVID-19 mortality $(4 / 4 ; 100 \%)$ than those without diabetes (0/10; 0\%; (OR, 90.71; 95\% Cl, 5.66-infinite; $P=0.0005)$. These findings suggest that Black SCT carriers with preexisting diabetes are at disproportionally higher risk for COVID-19 mortality. Confirmation by larger studies is warranted.
\end{abstract}

\section{INTRODUCTION}

The coronavirus (COVID-19) pandemic has impacted communities worldwide, but its effects are disproportionately expressed in certain groups. The Center for Disease Control reported that non-Hispanic Black individuals are approximately 1.4-times and 2.8-times more likely to test positive for and die of COVID-19, respectively, compared with White individuals. ${ }^{1}$ This finding is supported by United Kingdom Biobank (UKB) studies, which found significantly higher positive rates among Black individuals even after adjusting for biological, behavioral, and socioeconomic factors. ${ }^{2,3}$ Additionally, evidence shows that individuals with chronic conditions such as cardiovascular disease, diabetes, chronic respiratory disease, hypertension, and sickle cell disease (SCD) are particularly vulnerable to more severe COVID-19 disease. ${ }^{1,4}$

The intersection of race and SCD makes it important to consider their impact on COVID-19. SCD is a genetic disorder whereby patients inherit two pathogenic mutations of the gene that codes for hemoglobin ( $\mathrm{Hgb})$. It mostly affects people of sub-Saharan African descent; therefore, it is common in many Black communities worldwide.,6 Although SCD patients are relatively rare, individuals with only one pathogenic mutation, called sickle cell trait (SCT) carriers, are more common. Compared with SCD, which is associated with serious health conditions, ${ }^{6-9}$ most SCT carriers are considered asymptomatic. However, approximately $40 \%$ of the $\mathrm{Hgb}$ in carriers can induce sickling under certain conditions. ${ }^{5,6}$ Additionally, recent evidence suggests that $\mathrm{Hgb}$ may also have a modulatory role in the immune response against RNA viruses, including severe acute respiratory syndrome coronavirus 2 (SARS-CoV-2). ${ }^{10,11}$

* Address correspondence to Jianfeng Xu, NorthShore University HealthSystem, 1001 University Place, Evanston, IL 60201. E-mail: jxu@northshore.org
Because of the novelty of the COVID-19 pandemic, it is still unknown how SCT carriers are uniquely affected by the virus because of defective Hgb. The larger number of carriers compared with SCD patients makes studying this condition more relevant to a larger population and more feasible (increased statistical power from larger sample sizes). Therefore, the goal of this study was to examine the association of COVID-19 and SCT carriers. Based on the role of Hgb in immune responses and higher risk for COVID-19 among Black individuals, we hypothesized that SCT was associated with an increased risk for COVID-19 and mortality, especially for Black individuals. Furthermore, we hypothesized that other candidate genetic and lifestyle risk factors as well as comorbidities may modify the risk of COVID-19 for SCT carriers.

\section{METHODS}

This study was performed using the UKB, a populationbased study with extensive genetic and phenotypic data for approximately 500,000 individuals cross the United Kingdom 40 to 69 years of age at recruitment. ${ }^{12}$ Extensive phenotypic and health-related information was available for each participant, including questionnaire answers, biological measurements, lifestyle indicators, and biomarkers in blood and urine. Follow-up information was provided by linking health and medical records. Candidate comorbidities and risk factors [body mass index (BMI), diabetes, chronic obstructive pulmonary disease, hypertension, cancer, stroke, and smoking status] were obtained through the International Classification of Diseases-10 (ICD-10) codes and self-report.

The UKB Axiom SNP array data were available for all subjects, whereas whole-exome sequencing (WES) data were available for approximately $40 \%$ of subjects. For subjects without WES data, SCT carriers were identified based on ICD codes alone (i.e., D57.3). Codes for SCD (D57.0, D57.1, D57.2, and D57.8) and $\beta$-thalassemia (D56.1) were excluded. 
TABLE 1

Association of SCT with COVID-19 positivity and mortality in the UK Biobank

\begin{tabular}{|c|c|c|c|c|c|c|c|c|}
\hline & \multirow[b]{2}{*}{ Subjects } & \multirow{2}{*}{$\begin{array}{l}\text { No. (\%) tested for } \\
\text { COVID-19 }\end{array}$} & \multicolumn{3}{|c|}{ Positive for COVID-19 } & \multicolumn{3}{|c|}{ Died of COVID-19 } \\
\hline & & & Subjects & OR $(95 \% \mathrm{Cl})^{*}$ & $P$ value* & Subjects & OR $(95 \% \mathrm{Cl})^{*}$ & $P$ value* \\
\hline All & 500,822 & 44,724 (8.9\%) & 7,849 (17.5\%) & & & 495 (6.3\%) & & \\
\hline \multicolumn{9}{|l|}{ By race } \\
\hline White individuals & 471,180 & 41,660 (8.8\%) & 7,167 (17.2\%) & Ref* & & 445 (6.2\%) & Ref* & \\
\hline Black individuals & 8,017 & $862(10.8 \%)$ & $181(21 \%)$ & $0.99(0.84-1.18)$ & 0.95 & 25 (13.8\%) & $6.3(3.76-10.25)$ & $5.19 \mathrm{E}-13$ \\
\hline \multicolumn{9}{|l|}{ By SCT status } \\
\hline No SCT & 500,093 & $44,631(8.9 \%)$ & 7,828 (17.5\%) & Ref† & & $491(6.3 \%)$ & Ref† & \\
\hline SCT & 729 & $93(12.8 \%)$ & $21(22.6 \%)$ & $1.12(0.66-1.84)$ & 0.67 & $4(19 \%)$ & $2.87(0.69-9.95)$ & 0.11 \\
\hline \multicolumn{9}{|l|}{ Black individuals } \\
\hline No SCT & 7,446 & 791 (10.6\%) & 167 (21.1\%) & Ref† & & $21(12.6 \%)$ & Ref $^{*}$ & \\
\hline SCT & 571 & 71 (12.4\%) & $14(19.7 \%)$ & $0.98(0.51-1.78)$ & 0.96 & $4(28.6 \%)$ & $3.04(0.69-11.82)$ & 0.12 \\
\hline
\end{tabular}

$\mathrm{Cl}=$ confidence interval; $\mathrm{OR}=$ odds ratio; $\mathrm{SCT}=$ sickle cell trait.

* Standard logistic regression analysis adjusted for age at COVID-19 test and sex.

† Standard logistic regression analysis adjusted for age at COVID-19 test, sex, and race.

For subjects with WES data, carriers were identified using a combination of ICD codes and heterozygous Glu6Val mutations in the $H B B$ gene. Subjects with ICD codes for SCD, homozygous Glu6Val, or double heterozygous (heterozygous Glu6Val mutation plus any known mutations in $H B B$ such as Glu121Lys, Glu121Gln, Asp73Asn, and Val23lle) were considered to have SCD and removed from the remaining analyses. ${ }^{13}$ Blood types (ABO) were inferred from rs8176746 and rs8176719 genotypes, ${ }^{14}$ and APOE alleles were inferred from rs7412 and rs429358 genotypes ( $\varepsilon 2=\mathrm{T} / \mathrm{T}$, $\varepsilon 3=\mathrm{C} / \mathrm{T}$, and $\varepsilon 4=\mathrm{C} / \mathrm{C}){ }^{15}$

The COVID-19 test results for the UKB participants were provided by Public Health England (for participants residing in England). This information was updated weekly. Additional COVID-19 data are also collected through general practitioner (primary care) data, hospital inpatient data, death data, and critical care data (for COVID-19-positive patients); these data are updated monthly.

Logistic regression analysis with the maximum likelihood estimation method was used to test the associations of SCT and COVID-19 positivity/mortality and adjusted for other known demographic variables (race, age at testing, BMI, and sex). An exact logistic regression analysis was used when the sample size was small (total sample size $<40$ ); we modeled the log odds of binary outcomes as a linear combination of the predictor variables. The estimates provided by exact logistic regression do not depend on asymptotic results caused by small cells. The model diagnosis and goodness of fit were examined using Hosmer and Lemeshow's test. Specific models used for each test are described in each table.

\section{RESULTS AND DISCUSSION}

Based on the ICD codes and mutations in the $H B B$ gene, 729 subjects recorded in the UKB were classified as SCT carriers. Consistent with previous reports, ${ }^{5,6}$ most of the carriers were Black (571/729; 78\%). The estimated carrier rate by race was highest for Black individuals $(571 / 8,017 ; 7.1 \%)$ and considerably lower for other races (Asian, 11/11,399 [0.1\%]; White, 20/471,180 [0.004\%]; mixed race, 40/2,928 [1.4\%]; and other, 69/4,605 [1.5\%]). It was noted that the carrier rate was significant higher for females, especially those younger than 45 years at the time of recruitment. For Black individuals, for example, the carrier rate was $7.8 \%$ $(360 / 4,633)$ for females and $6.2 \%(211 / 3,384)$ for males overall ( $P=0.009)$; however, it was $8.9 \%(82 / 920)$ for females and $4.6 \%(36 / 783)$ for males among subjects younger than 45 years at the time of recruitment $(P=0.0007)$ (Supplemental Table 1). This novel observation warrants confirmation and may suggest higher mortality for men at younger ages.

By December 21, 2020, COVID-19 test results were available for 44,724 subjects (approximately 8.9\% [44,724/500,822] of the UKB population). Among those tested for COVID-19, $17.5 \%(7,849 / 44,724)$ had positive results; of these, $6.3 \%$ $(495 / 7,849)$ died of COVID-19 (Table 1). Black individuals had nonsignificantly higher COVID-19 positivity rates (21.0\% [181/ 862] compared to $17.2 \%[7,167 / 41,660] ; P=0.94$ ) but significantly higher COVID-19 death rates than White individuals (13.8\% [25/181] compared to 6.2\% [445/7,167]; $P<0.0001)$. The mean age at the time of the COVID-19 diagnosis was also significantly younger for Black individuals (64.5 years) than for White individuals (69.7 years) $(P<0.0001)$. Similarly, the mean age at the time of death attributable to COVID-19 was significantly younger for Black individuals (71.1 years) than for White individuals (75.1 years) $(P<0.0001)$. Most of these findings are consistent with those of other published studies. ${ }^{2,3}$

Compared with individuals who were not SCT carriers, SCT carriers had similar COVID-19 positivity rates but higher COVID-19 mortality rates overall among Black individuals (Table 1). For Black individuals, the positivity rates were $19.7 \%$ (14/71) and $21.1 \%$ (167/791) for subjects with SCT and without SCT $(P=0.96)$. The death rate was higher, but not statistically significant, for Black SCT carriers $(4 / 14 ; 28.6 \%)$ than for Black noncarriers of SCT $(21 / 167 ; 12.6 \%)$ (odds ratio [OR], 3.04; 95\% confidence interval [Cl], 0.69-11.82; $P=0.12$ ).

We also examined whether other candidate demographic and genetic factors as well as comorbidities affected COVID-19 positivity and mortality of Black SCT carriers $(N=$ 71). ${ }^{1,2,4,15}$ Most of these variables were not significantly associated with COVID-19 positivity and mortality (Table 2), partially because of the small sample size. However, comorbid diabetes (type 1 diabetes and type 2 diabetes) was more common in SCT carriers who were positive for COVID-19 (4/ $14 ; 28.6 \%)$ than in SCT carriers who were negative for COVID-19 (7/57; 12.3\%) (OR, 2.81; 95\% CI, 0.51-13.80; $P=$ $0.21)$. In particular, diabetes was significantly more common in SCT carriers who died of COVID-19 $(4 / 4 ; 100 \%)$ than in those who survived (0/10; 0\%) (OR, 90.71; $95 \% \mathrm{Cl}$, 5.66-infinite; $P=0.0005)$. 


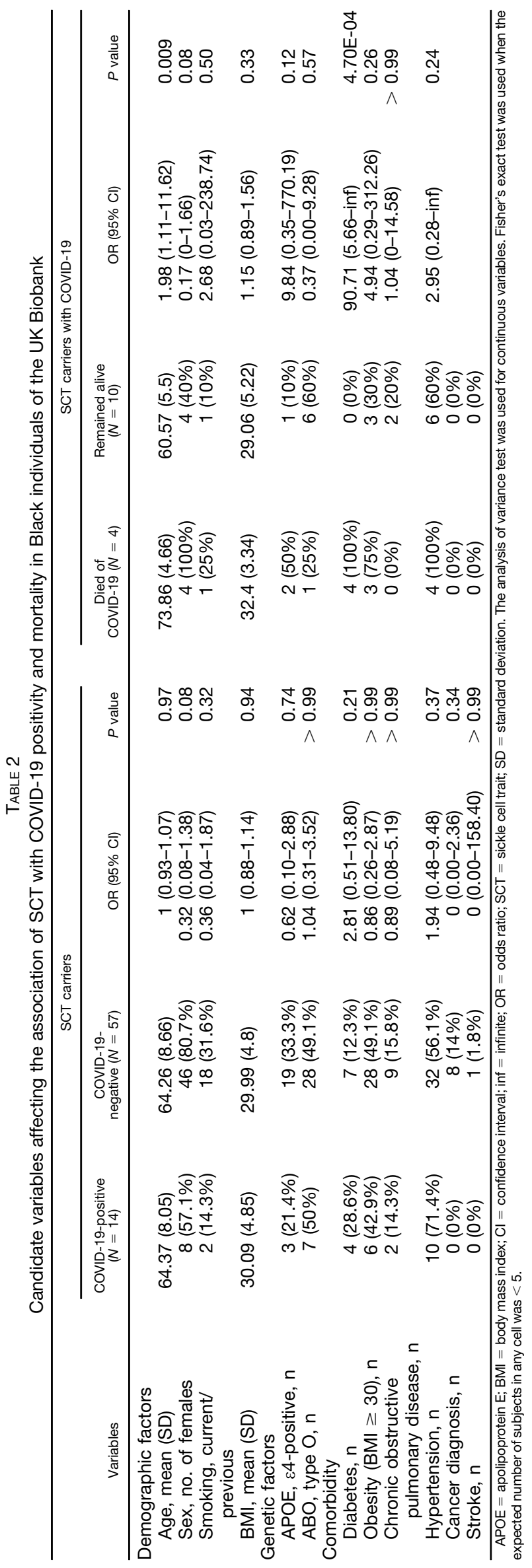

The effect of diabetes comorbidity on COVID-19 has been reported previously. ${ }^{1,4}$ During the current study, we found diabetes was significantly associated with a higher risk of death cause by COVID-19 (OR, 1.75; 95\% Cl. 1.32-2.31; $P<0.0001$ ) (Table 3). The risks for COVID-19 positivity and mortality were higher for Black diabetes patients, and especially for Black SCT carriers. For example, all four Black SCT carriers died of COVID-19, but 6 of 34 (17.6\%) Black noncarriers of SCT died of COVID-19 (OR, 9.02; 95\% Cl, 0.74 -infinite; $P=0.05$ ).

A unique strength of this study was our better ability to identify SCT carriers in the population using genetic information. We found that SCT is considerably under-reported by ICD codes. Among subjects with WES data in our study, we found the majority of SCT carriers defined by heterozygous Glu6Val did not have an ICD code for SCT (409/527; 77.6\%). This finding has two important implications. First, many SCT carriers may be misclassified if they are without WES data, which would create a bias toward a null finding. Second, and probably more important, our data suggest that the SCT carrier rate is considerably higher than that previously estimated, and the majority of SCT carriers in the general population are not aware of their status. Therefore, many SCT carriers miss the opportunity for extra care during the prevention and management of COVID-19.

One limitation of this study was the relatively small sample size. However, this study represents one of the largest available studies of the association between COVID-19 and SCD/SCT. ${ }^{7-9}$ Confirmation of our findings by additional study populations is needed. Another limitation was the lack of detailed clinical information regarding COVID-19 symptoms and treatment. This limited our ability to perform a more comprehensive analysis. Nevertheless, because of the novel and emergent status of this pandemic, more data will likely become available for additional analyses by future studies to address both limitations of this study.

In conclusion, in this first study testing the association of SCT and COVID-19 using a population-based cohort, we found that Black SCT carriers had a trend of higher, albeit not statistically significant, COVID-19 mortality than noncarriers of SCT. We also found that Black SCT carriers with preexisting diabetes had significantly higher COVID-19 mortality than those without diabetes. Considering that the majority of SCT carriers may not be aware of their condition, and considering that Black individuals are already at disproportionally higher risk for both COVID-19 and diabetes, ${ }^{16}$ these findings suggest special care during the prevention and management of COVID-19 should be considered for Black SCT carriers.

Received December 29, 2020. Accepted for publication April 26, 2021.

Published online June 15, 2021

Note: Supplemental table appears at www.ajtmh.org.

Acknowledgments: We are grateful to the Ellrodt-Schweighauser, Chez, and Melman families for establishing Endowed Chairs of Cancer Genomic Research and Personalized Prostate Cancer Care at NorthShore University HealthSystem in support of Dr. Xu and Dr. Helfand, and the Rob Brooks Fund for Personalized Prostate Cancer Care at NorthShore University HealthSystem. The American Society of Tropical Medicine and Hygiene has waived the Open Access fee for this article due to the ongoing COVID-19 pandemic. 
TABLE 3

Sequential stratified association tests for COVID-19 positivity and mortality with diabetes, race, and SCT in the UK Biobank

\begin{tabular}{|c|c|c|c|c|c|c|c|c|}
\hline & \multirow[b]{2}{*}{ Subjects } & \multirow{2}{*}{$\begin{array}{l}\text { Subjects (\%) tested } \\
\text { for COVID-19 }\end{array}$} & \multicolumn{3}{|c|}{ Positive for COVID-19 } & \multicolumn{3}{|c|}{ Died of COVID-19 } \\
\hline & & & Subjects (\%) & OR $(95 \% \mathrm{Cl})$ & $P$ value & Subjects (\%) & OR (95\% Cl) & $P$ value \\
\hline \multicolumn{9}{|l|}{ By diabetes status } \\
\hline No diabetes & 469,225 & $40,371(8.6 \%)$ & $7,114(17.6 \%)$ & Ref* & & 379 (5.3\%) & Ref* & \\
\hline Diabetes & 31,597 & 4,353 (13.8\%) & 735 (16.9\%) & $0.98(0.9-1.07)$ & 0.69 & $116(15.8 \%)$ & $1.75(1.32-2.31)$ & 7.78E-05 \\
\hline \multicolumn{9}{|c|}{ By race with diabetes } \\
\hline White individuals & 27,683 & 3,771 (13.6\%) & 599 (15.9\%) & Ref† & & 95 (15.9\%) & Ref† & \\
\hline Black individuals & 978 & 156 (16.0\%) & $38(24.4 \%)$ & $1.71(1.15-2.49)$ & $6.10 \mathrm{E}-03$ & $10(26.3 \%)$ & $3.92(1.62-9.07)$ & 1.67E-03 \\
\hline \multicolumn{9}{|c|}{ By SCT with diabetes for Black individuals } \\
\hline No SCT & 895 & $145(16.2 \%)$ & $34(23.4 \%)$ & Ref $†$ & & $6(17.6 \%)$ & Refł & \\
\hline SCT & 83 & $11(13.3 \%)$ & $4(36.4 \%)$ & $3.2(0.74-12.74)$ & 0.1 & $4(100 \%)$ & 9.02 (0.74-inf) & 0.05 \\
\hline
\end{tabular}

$\mathrm{APOE}$ = apolipoprotein $\mathrm{E} ; \mathrm{BMI}$ = body mass index; $\mathrm{Cl}$ = confidence interval; inf = infinite; $\mathrm{OR}$ = odds ratio; SCT = sickle cell trait; $\mathrm{SD}=$ standard deviation.

* Standard logistic regression analysis adjusted for age at COVID-19 test, sex, BMI, and race.

† Standard logistic regression analysis adjusted for age at COVID-19 test, BMl, and sex.

$\ddagger$ Exact logistic regression analysis adjusted for age at COVID-19 test and sex.

Disclosure: All authors declare no support from any organization for the submitted work; no financial relationships with any organizations that might have an interest in the submitted work in the previous three years; no other relationships or activities that could appear to have influenced the submitted work. The UKB was approved by North West-Haydock Research Ethics Committee (REC reference: 16/NW/0274; IRAS project ID: 200778). UKB data were accessed through a Material Transfer Agreement under Application Reference Number 50295. This study was performed in accordance with the Declaration of Helsinki. All UKB participants provided their informed consent before any data/samples were collected. The data used in this study are available in the UK Biobank, a publicly available repository. Data were accessed through a Material Transfer Agreement under Application Reference Number 50295. For additional information, please feel free to contact the corresponding author.

Authors' addresses: W. Kyle Resurreccion, Zhuqing Shi, Jun Wei, Chi-Hsiung Wang, Rong Na, S. Lilly Zheng, Brian T. Helfand, and Jianfeng Xu, Program for Personalized Cancer Care, NorthShore University HealthSystem, Evanston, IL, E-mails: wresurreccion@ northshore.org, shizhuqing1991@hotmail.com, weijun19992019@ gmail.com, cwang@northshore.org, narong.hs@gmail.com, szheng@ northshore.org, brianhelfand@gmail.com, and jxu@northshore.org. Joseph Hulsizer, Lake Forest Academy, Lake Forest, IL, E-mail: joerig4@gmail.com. Clay Struve, CSS LLC, Chicago, IL, E-mail: clay@csstrading.com. Brian T. Helfand and Jianfeng Xu, Department of Surgery, University of Chicago Pritzker School of Medicine, Chicago, IL, E-mails: brianhelfand@gmail.com and jxu@northshore. org. Janardan Khandekar, Center for Molecular Medicine, NorthShore University HealthSystem, Evanston, IL, E-mail: jkhandekar@northshore.org. Liana K. Billings, Department of Medicine, NorthShore University HealthSystem, Evanston, IL, and Department of Medicine, University of Chicago Pritzker School of Medicine, Chicago, IL, E-mail: Ibillings@northshore.org. Michael S. Caplan, Department of Pediatrics, NorthShore University HealthSystem, Evanston, IL, E-mail: mcaplan@northshore.org.

This is an open-access article distributed under the terms of the Creative Commons Attribution (CC-BY) License, which permits unrestricted use, distribution, and reproduction in any medium, provided the original author and source are credited.

\section{REFERENCES}

1. NCIRD, 2020. COVID-19: People at Increased Risk. Available at: https://www.cdc.gov/coronavirus/2019-ncov/need-extraprecautions/index.html. Accessed December 28, 2020.
2. Kolin DA, Kulm S, Christos PJ, Elemento O, 2020. Clinical, regional, and genetic characteristics of COVID-19 patients from UK Biobank. PLoS One 15: e0241264.

3. Niedzwiedz CL et al., 2020. Ethnic and socioeconomic differences in SARS-CoV-2 infection: prospective cohort study using UK Biobank. BMC Med 18: 160.

4. Jordan RE, Adab P, Cheng KK, 2020. COVID-19: risk factors for severe disease and death. BMJ 368: $\mathrm{m} 1198$.

5. Piel FB et al., 2013. Global epidemiology of sickle haemoglobin in neonates: a contemporary geostatistical model-based map and population estimates. Lancet 381: 142-151.

6. Steinberg $\mathrm{MH}, 1999$. Management of sickle cell disease. $N$ Engl J Med 340: 1021-1030.

7. Sahu KK, Siddiqui AD, Cerny J, 2020. Managing sickle cell patients with COVID-19 infection: the need to pool our collective experience. $\mathrm{Br} \mathrm{J}$ Haematol 190: e86-e89.

8. Oualha $M$ et al., 2020. Severe and fatal forms of COVID-19 in children. Arch Pediatr 27: 235-238.

9. Arlet JB et al., 2020. Prognosis of patients with sickle cell disease and COVID-19: a French experience. Lancet Haematol 7: e632-e634.

10. Yang Q, Bai SY, Li LF, Li S, Zhang Y, Munir M, Qiu HJ, 2019. Human hemoglobin subunit beta functions as a pleiotropic regulator of RIG-I/MDA5-mediated antiviral innate immune responses. J Virol 93: e00718-e00719.

11. Brenner SR, 2021. Erythropoietin-induced hemoglobin subunit beta may stimulate innate immune RNA virus pattern recognition, suppress reactive oxygen species, reduce ACE2 viral doorway opening, and neutrophil extracellular traps against COVID-19. J Med Virol 93: 180-181.

12. Bycroft $\mathrm{C}$ et al., 2018. The UK Biobank resource with deep phenotyping and genomic data. Nature 562: 203-209.

13. Rees DC, Williams TN, Gladwin MT, 2010. Sickle-cell disease. Lancet 376: 2018-2031.

14. Melzer $D$ et al., 2008. A genome-wide association study identifies protein quantitative trait loci (pQTLs). PLoS Genet 4: e1000072.

15. Kuo CL, Pilling LC, Atkins JL, Masoli JAH, Delgado J, Kuchel GA, Melzer D, 2020. APOE e4 genotype predicts severe COVID-19 in the UK Biobank community cohort. J Gerontol A Biol Sci Med Sci 75: 2231-2232.

16. Menke A, Casagrande S, Geiss L, Cowie CC, 2015. Prevalence of and trends in diabetes among adults in the United States, 1988-2012. JAMA 314: 1021-1029. 